\title{
Macht in der Lehre der Friedens- und Konfliktforschung
}

\section{Einleitung}

Als Untersuchungsgegenstand spielt die Kategorie Macht eine zentrale Rolle in der Lehre der Friedens- und Konfliktforschung. Machtmissbrauch kann eine Ursache gewaltsamer Konflikte sein, Machthierarchien wirken sich auf den Austrag von Konflikten aus, und die Ausbalancierung von Ungleichheiten spielt eine wesentliche Rolle für die Bearbeitung von gewaltsamen Konflikten. Mit der Tatsache, dass Machtverhältnisse auch die Beziehung zwischen Lehrenden und Studierenden prägen und somit im disziplinären Lehralltag allgegenwärtig sind, hat sich die Friedensund Konfliktforschung bisher hingegen nicht auseinandergesetzt. Studierende sollen zwar Kompetenzen erwerben, die sie zur konstruktiven Arbeit in Konflikten ermächtigen; eine Reflektion darüber, wie Machtasymmetrien diesen Lernprozess behindern können, steht jedoch noch aus. Auch die hochschuldidaktische Literatur beschäftigt sich kaum mit diesem Phänomen. Dieser Beitrag setzt an diesem blinden Fleck an und erörtert, inwiefern Macht in der Hochschullehre eine Rolle spielt. Worauf beruht die Macht von Lehrenden? Wie beeinflussen Machtfragen Lernprozesse? Inwieweit lassen sich Machtasymmetrien mit den Zielen der Friedens- und Konfliktforschung vereinbaren? Und schließlich: Gibt es ein vertretbares und richtiges Maß an Machtorientierung und falls ja, wie kann es bestimmt werden? ${ }^{1}$

\section{Macht und Machtunterschiede in der Hochschullehre}

Max Weber definiert Macht als »Chance, innerhalb einer sozialen Beziehung den eigenen Willen auch gegen Widerstreben durchzusetzen, gleichviel worauf diese Chance beruht « (Weber 1976: 28). Demnach kann Macht eingesetzt werden, sie muss aber nicht genutzt werden (»Chance«), ihr Gebrauch ist intentional (»eigener Wille«), die Folgsamkeit kann gegen den Willen des Betroffenen, aber auch freiwillig erfolgen (»auch gegen Widerstand«) und die Macht kann auf ganz unterschiedlichen Grundlagen beruhen (»gleichviel worauf diese Chance beruht«) (Crott 1997: 231). Zwar ist diese Definition besonders auf das Phänomen politischer Macht

1 Der Ursprung dieses Beitrags geht zurück auf das AFK-Kolloquium 2011 und das dort angebotene Panel »Macht und Konflikte in der Hochschullehre«. Die zentralen Ideen und Argumente hierfür haben Verena Brenner und ich gemeinsam entwickelt, sie hat daher wesentlich zu diesem Artikel beigetragen. 
ausgerichtet, doch auch sozialpsychologische Definitionen, wie sie für die Betrachtung von Lehr-Lern-Situationen angemessener erscheinen mögen, unterscheiden sich hiervon kaum (vgl. Witte 2001: 6-11): »Unter sozialer Macht verstehen wir [...] die aufgrund ihrer Verfügungsgewalt über Ressourcen von den Partnern zugeschriebene Fähigkeit von Personen oder Gruppen, auf kognitive oder Verhaltensaspekte dieser Partner einzuwirken« (Schneider 1977: 35, zit. nach Witte 2001: 7). Macht ist in zwischenmenschlichen Beziehungen allgegenwärtig und ein wechselseitiges Phänomen. In der Regel zeichnen sich Machtverhältnisse jedoch durch eine Asymmetrie aus. Dies zeigt auch der Blick auf das Verhältnis zwischen Lehrenden und Studierenden. ${ }^{2}$ Im Folgenden argumentiere ich, dass Machtungleichgewichte in der Lehre durch die strukturelle Beziehung Dozierende - Studierende und die damit verbundene Rollenverteilung gegeben sind und sich aus einer unterschiedlichen Verfügbarkeit von Machtressourcen ergeben. Darüber hinaus zeige ich auf, dass sich Macht in Lehrsituationen in ganz unterschiedlichen Formen manifestieren kann. Wie stark die Machtorientierung von Lehrenden ist, hängt wiederum von individuellen und strukturellen Kontextfaktoren ab.

\subsection{Machtunterschiede: Rollenverteilung und Ressourcen der Macht}

Machtunterschiede in der Lehre sind strukturell bedingt und ergeben sich aus der Position und sozialen Beziehung zwischen Dozierenden und Studierenden. Diese sozialen Strukturen haben Einfluss auf Identitäten, Interessen und Ressourcen (Barnett/Duvall 2005: 18-20). Die Lehrenden sind - so zumindest die Erwartungen diejenigen, die aufgrund ihres Wissens- und Kompetenzvorsprungs die Studierenden anleiten, unterrichten, prüfen oder sogar erziehen. Im Gegenzug werden Studierende als Lernende betrachtet, die an der Universität ihr Wissen vergrößern oder eine Berufsqualifikation erwerben. Eine hierarchische Positionierung wird auch bei der Betrachtung der Rollenverteilung deutlich. Dozierende, die dem Anspruch einer guten Lehre gerecht werden wollen, sind je nach Situation Experte, Moderator, Prüfer, Berater oder auch Vorbild (Winteler 2005: 24). Die Rolle des Prüfers setzt dabei eine Machtasymmetrie geradezu voraus, der Rolle des Experten ist sie inhärent. Andere Rollen, wie die des Beraters und des Moderators, lassen sich angemessener ausfüllen, wenn die Machtasymmetrien geringer sind und Lehrende auf den ostentativen Einsatz ihrer Machtmittel verzichten. Stellt man den situativen Rollen von Dozierenden das jeweilige Rollenpendant auf studentischer Seite ent-

2 Dieser Beitrag diskutiert die Machtbeziehungen zwischen Dozierenden und Studierenden. Darüber hinaus gibt es auch Machtungleichgewichte zwischen Studierenden, die sich auf Lernprozesse negativ auswirken können. 
gegen, so ergeben sich zumeist ungleiche Paarungen: Experte und Laie, Prüfer und Prüfling, Ratgeber und Ratsuchender, Vorbild und Nachahmer, Moderator und Diskutierende. ${ }^{3}$

Machtunterschiede zwischen Lehrenden und Studierenden beruhen jedoch nicht nur auf der sozialen Positionierung dieser Akteure im Setting Universität, sondern lassen sich auch durch die unterschiedliche Verfügbarkeit von Machtressourcen erklären (für eine Systematisierung von Grundlagen der Macht vgl. French/Raven 1959). Die Macht von Lehrenden beruht einerseits auf den Möglichkeiten der Belohnung oder Bestrafung von Studierenden. Darunter fallen Notenvergabe oder Empfehlungsschreiben. Weitere Beispiele sind die Übernahme von Betreuungsarbeiten, die Zulassung zu Exkursionen, das Anbieten von studentischen Hilfskraftstellen. Legitimation ist eine weitere Machtressource. Einflussnahme ist demnach möglich, weil sie als rechtmäßig angesehen wird. Dieser Aspekt ist eng mit der Rollenzuweisung Dozent verbunden: Es wird als sozial angemessen erachtet, dass Dozierende Regeln setzen, Prüfungen erstellen, Inhalte vorgeben, Diskussionen leiten, Redebeiträge bewerten. Mit Legitimation eng verbunden sind auch Faktoren wie Status und Ansehen, die auf akademischen Titeln, Auszeichnungen, dem Akquirieren von Drittmitteln oder Ähnlichem beruhen. Expertise als Machtbasis beruht auf dem Wissensvorsprung von Lehrenden. Zuletzt kann auch Identifikation eine Grundlage der Macht von Dozenten sein. Das ist dann der Fall, wenn Lehrende als Vorbilder wahrgenommen werden, auf deren Anerkennung Studierende besonderen Wert legen oder die sie nachahmen.

Betrachtet man die Machtressourcen von Studierenden, so fällt auf, dass diese geringer und häufig informell sind. Belohnung oder Bestrafung kann in Form von Evaluationen oder aber durch soziale Affirmation oder Missachtung ausgedrückt werden. Auch Legitimation spielt eine Rolle, da es sozial anerkannt ist, dass Studierende gewisse Leistungen von Seiten ihrer Lehrenden erwarten können (z.B. Begründung von Bewertungen). ${ }^{4}$ Identifikation und Expertise als Grundlagen studentischer Macht sind hingegen eher situativ bedingt und personenabhängig.

3 In der Realität sind diese Rollenverteilungen weniger fix und eindeutig als hier dargestellt. Beispielsweise können Studierende, die ein Referat halten, durchaus eine Expertenrolle einnehmen. Denkbar sind auch wechselseitige Beratung oder die Übernahme von Moderationsaufgaben durch Studierende.

4 Die Macht von Studierenden gegenüber Lehrenden manifestiert sich besonders, wenn diese als Gruppe sprechen - indem sie zum Beispiel bestimmte Forderungen stellen oder Kritik üben. Hier zeigt sich eine Nähe zum Machtbegriff von Hannah Arendt, die Macht als »menschliche[n] Fähigkeit, nicht nur zu handeln oder etwas zu tun, sondern sich mit anderen zusammenzuschließen und im Einvernehmen mit ihnen zu handeln« (Arendt 2000: 45) definiert. 


\subsection{Wie Macht in der Lehre eine Rolle spielen kann}

Machtressourcen müssen nicht genutzt werden, sondern stellen ein Potenzial dar. Dozenten können die ihnen zur Verfügung stehenden Machtmittel, sofern sie sich ihrer bewusst sind und diese reflektieren, zu einem großen Teil flexibel einsetzen. Diese Varianz in der Manifestation von Macht drückt sich unter anderem im Umgang mit folgenden Aspekten aus:

- Mitgestaltung: Wer bestimmt die Lerninhalte? Wie und durch wen werden Arbeitsformen, Art und Umfang der Leistungsnachweise, Seminarregeln und Ähnliches bestimmt?

- Verantwortung und Autonomie: Werden Lern- und Arbeitsprozesse durch Vorgaben strukturiert oder können sie von den Studierenden autonom gestaltet werden? Steht das Wissen des Lehrenden im Mittelpunkt oder werden Studierende dazu ermutigt, sich ihre eigenen Sinnstrukturen zu konstruieren? Kümmert sich der Dozent um alles oder werden Aufgaben an Studierende abgegeben (Moderation von Diskussionen, Vorbereitung von Exkursionen etc.)?

- Kommunikation: Wie sind die Redeanteile zwischen Lehrenden und Studierenden verteilt? Wie wird gesprochen: Werden Fachbegriffe erläutert? Ist die Sprache allgemein verständlich oder mit Fremdworten gespickt und somit exkludierend? Ist das sprachliche Verhalten reversibel, d.h. sprechen die Dozierenden mit den Studierenden so wie diese auch mit ihnen reden können? Ist die Sprache wertschätzend oder abwertend?

- Feedback: Wird Feedback gegeben? In welcher Form findet diese Rückmeldung statt (Balance zwischen Würdigung und Kritik, Klarheit der Formulierungen, Wertung oder Beschreibung)? Gibt nur der Dozent Feedback oder auch Studierende? Bekommen nur die Studierenden Feedback oder auch der Lehrende?

- Umgang mit Regeln: Gibt es Regeln? Werden diese begründet? Gelten Regeln wechselseitig oder nur für Studierende? Werden Regeln durchgesetzt? Wie strikt ist die Regeldurchsetzung? Wie transparent sind Entscheidungen zum Umgang mit Regeln?

- Prüfungen: Bereitet die Veranstaltung auf die Prüfung vor? Gibt es klare Erläuterungen, was in der Prüfung erwartet wird? Wie transparent und begründet ist die Notengebung? Werden Prüfungen zur Disziplinierung eingesetzt?

\subsection{Kontextfaktoren: Was beeinflusst den Grad der Machtorientierung?}

Sowohl Machtressourcen als auch der konkrete Einsatz von Macht sind von einer Reihe von Kontextfaktoren abhängig. So sind die oben dargestellten Machtressourcen (Belohnung, Bestrafung, Legitimation, Expertise und Identifikation) keine ab- 
soluten, gegebenen Größen. Belohnung und Bestrafung hängen vom Grad der Abhängigkeit der Studierenden ab und werden beeinflusst von Aspekten wie beispielsweise: Wie stark fließt eine Seminarnote in das Examen ein? Wie wichtig ist ein erfolgreicher Abschluss für die berufliche Karriere? Stehen andere Dozenten als Betreuer zur Verfügung? Die Machtfülle eines Dozenten hängt zudem mit individuellen Qualitäten zusammen wie Selbstsicherheit und Auftreten. Schließlich spielen auch perzeptive Faktoren eine große Rolle: Eine Machtressource wie Wissen wird beispielsweise erst dadurch relevant, dass die Expertise des Lehrenden von den Studierenden wahrgenommen und anerkannt wird. Ähnlich bedeutsam ist Perzeption für die Machtquellen Legitimation und Identifikation.

Wie sehr Machtressourcen tatsächlich genutzt werden und wie machtorientiert das Verhalten eines Dozenten ist, hängt von dessen Selbstverständnis (welche der Rollen eines Dozierenden sind ihm besonders wichtig?) sowie seinen Konzeptionen von Lernen und Lehren ab. Ferner gibt es strukturelle Zwänge, die den Einsatz von Macht bedingen. Hierzu zählen vor allem die gesellschaftlichen Ansprüche an die Institution Universität. Diese ist nicht nur dafür zuständig, junge Menschen zu bilden und auszubilden, sondern soll darüber hinaus auch die Qualifikation und Eignung der Studienabgänger für bestimmte Tätigkeiten zertifizieren. Das Universitätsexamen dient demnach als Ausweis für die Eignung für bestimmte Aufgaben und als Eintrittskarte in die Berufswelt. Zudem wollen potenzielle Arbeitgeber wissen, wie gut ein Bewerber ist, was die Forderung nach einem Vergleich von Leistungen zur Folge hat. Die Gesellschaft verlangt also Leistungen wie Prüfungen, die einen Machteinsatz bedingen. Aber auch Modulhandbücher, Prüfungsordnungen, disziplinäre Lehrkulturen, Praktiken am Institut oder Erwartungshaltungen von Kollegen können als Zwänge empfunden werden, die eine bestimmte Form von machtorientierter Lehre bedingen. So können Lehrinhalte durch Modulhandbücher vorgegeben sein und damit die Mitbestimmungsmöglichkeiten für Studierende begrenzen. Ein anderes Beispiel ist die Vorschrift von Klausuren in der Prüfungsordnung, was den Einsatz von Prüfungsformen erschwert, die selbstbestimmtes Lernen begünstigen.

\section{Macht und Machtasymmetrien bewertet: Die Blickwinkel von Lernpsychologie, Hochschuldidaktik und Friedens- und Konfliktforschung}

Faktoren, die mit Machtausübung im Zusammenhang stehen, beeinflussen den Verlauf und Erfolg von Lernprozessen. Menschen lernen besser, wenn sie sich sicher und wertgeschätzt fühlen, sich als selbstwirksam erleben, autonom bestimmen können, mit welchen Themen sie sich beschäftigen wollen, und vor Herausforderungen 
gestellt werden, die sie bewältigen können. Werden die Herausforderungen hingegen als zu groß wahrgenommen oder ist das Lernen sehr fremdbestimmt, kann sich daraus ein negativer Lernkreislauf ergeben, der zu Angst, negativen Selbstzuschreibungen und einer Verstetigung von Misserfolgen führt (Spitzer 2007). Die Entstehung negativer Lernspiralen lässt sich dabei nicht grundsätzlich durch einen zu starken, sondern vielmehr durch einen unangemessenen Einsatz von Macht erklären. Überforderung kann beispielsweise die Folge von externem Druck sein. Sie kann aber auch die Folge von zu viel Autonomie sein, die dazu führt, dass Studierende sich orientierungslos fühlen. ${ }^{5}$ Von besonderer Bedeutung für den Lernerfolg sind ferner Emotionen und zwischenmenschliche Beziehungen - wenn Dozierende keine Wertschätzung ausdrücken oder Studierende sich angegriffen fühlen, wirkt sich dies negativ aus.

Die Hochschuldidaktik propagiert eine studentenzentrierte Lehre und einen Perspektivenwechsel vom Lehren zum Lernen. Kernaufgaben von Lehrenden sind demnach nicht mehr Instruktion und Vermittlung von Inhalten, sondern die Gestaltung von förderlichen Lernumgebungen und Lernberatung (Winteler 2002: 42-43; Wildt 2006). An die Stelle von Belehrung tritt Begleitung, Beurteilung wird durch Feedback ersetzt, der Hörsaal wird zur Werkstatt, in der das Problemlösen im Vordergrund steht (Blom 2000: 11-12). Hintergrund dieser Entwicklungen sind Erkenntnisse der konstruktivistischen Lernpsychologie, wonach Lernen ein selbstgesteuerter, aktiver und konstruktiver Prozess ist, der von außen allenfalls unterstützt werden kann (Siebert 2006). Folgt man diesen Ausführungen, so verlagert sich die Aktivität in der Lehre vom Dozierenden zu den Studierenden, die nicht mehr Wissensempfänger sind, sondern aktive und unabhängige Lernende. Machtasymmetrien zwischen Dozenten und Studierenden sind demnach dysfunktional und sollten abgebaut werden.

Speziell auf die Friedens- und Konfliktforschung bezogen, stellt sich zusätzlich die Frage, wie Machtungleichgewichte in der Lehre reflektiert werden. Gerade eine Disziplin, die Machtfragen einen zentralen Erklärungswert bei der Analyse von Konflikten zuweist, sollte mit Machtunterschieden, wie sie in der Lehre auftauchen, bewusst umgehen. Dies gilt umso mehr, als dass Machtasymmetrien sich auch auf die Ziele der Friedens- und Konfliktforschung auswirken. Ein Vergleich der verschiedenen friedenswissenschaftlichen Masterstudiengänge in Deutschland zeigt,

5 Diese Überforderung kann die Folge eines bewussten Machtverzichts von Lehrenden sein, die Studierenden die Verantwortung dafür überlassen möchten, mit welchen Themen sie sich beschäftigen, wie sie ihren Arbeitsprozess organisieren etc. Darüber hinaus kann eine solche Überforderung aber auch aus der Unwissenheit des Lehrenden oder seinem fehlenden Engagement resultieren - in diesem Fall steht das Verhalten in keinem Zusammenhang mit Machtfragen. 
dass Studierende nicht nur analytische Kompetenzen erwerben sollen, sondern dass Praxisorientierung zumeist eine große Rolle spielt. Zielsetzung der Masterangebote ist es, soziale Kompetenzen zu vermitteln und die Handlungsfähigkeit der Studierenden so zu fördern, dass sie zur friedlichen Bearbeitung von Konflikten beitragen können (Brühl et al. 2005). Nimmt man ferner die normative Orientierung der Friedens- und Konfliktforschung hinzu, die auf die Förderung von Frieden ausgerichtet ist, so geht es auch um eine Ermächtigung zur Einmischung in gesellschaftliche Diskurse oder um die Ermutigung, den Blick für das Visionäre, die Alternativen zur Gewalt, zu öffnen (Nielebock 2011). Eine starke Machtorientierung von Lehrenden steht diesen Zielen gleich in zweierlei Hinsicht entgegen: Zum einen lassen sich soziale Kompetenzen und Handlungsfähigkeit nicht vermitteln oder erklären, sondern müssen eingeübt werden und bedürfen somit einer Lernsituation, die durch Dialog, Offenheit, praktische Übungen und Eigenverantwortung geprägt ist. Zum anderen konterkariert eine Lehre, die durch ausgeprägte Formen der Machtausübung gekennzeichnet ist, die Inhalte einer auf die Förderung gewaltfreier Konfliktbearbeitung ausgerichteten Friedensforschung, da in einem solchen Fall - überspitzt formuliert - der Frieden mit den Mitteln von Zwang und Unterdrückung gelehrt wird.

\section{Die richtige Balance finden: Wie viel Machtorientierung ist sinnvoll?}

Die bisherigen Ausführungen lassen sich in drei Punkten zusammenfassen: (1) In der Hochschullehre sind Machtasymmetrien faktisch gegeben, ob gewollt oder nicht; (2) Das Ausmaß an Machtorientierung von Dozenten ist variabel und hängt von deren Rollen-, Lehr- und Lernverständnis ab; (3) Die Erkenntnisse von Hirnforschung, Lernpsychologie und Hochschuldidaktik weisen darauf hin, dass Machtasymmetrien für Lernprozesse eher problematisch sind und weniger meist mehr ist. Auch die Ziele der Friedens- und Konfliktforschung lassen sich durch eine Reduzierung von Machtungleichgewichten besser erreichen. Dennoch sei darauf hingewiesen, dass auch zu wenig Einflussnahme sich negativ auf Lehr-Lern-Situationen auswirken kann. Das kann sich in einem Fehlen von Strukturen und Regeln ausdrücken oder in studentischen Irritationen über ein als unangemessen empfundenes Rollenverhalten von Dozenten.

Da Macht also nicht negiert werden kann und angemessen eingesetzt werden muss, stellt sich die Frage nach dem richtigen Maß. Folgt man dem Modell der Wertequadrate (nach Paul Helwig, weiterentwickelt von Friedemann Schulz von Thun), dann hat jedes Verhalten nur dann einen konstruktiven Wert, wenn es sich in einem Spannungsverhältnis zu einem positiven Gegenwert befindet (Schulz von 
Thun 2008: 38). Für machtorientiertes Verhalten in der Lehre heißt das beispielsweise, dass es gut ist, wenn Dozierende Strukturen vorgeben. Diese Strukturierung sollte aber in Balance gehalten werden mit Flexibilität, ansonsten mutiert die Strukturgebung zur Rigidität. Flexibilität ohne Struktur hingegen wird zur Beliebigkeit oder zum konzeptlosen Laissez-faire.

Abbildung: Beispiel eines Wertequadrats

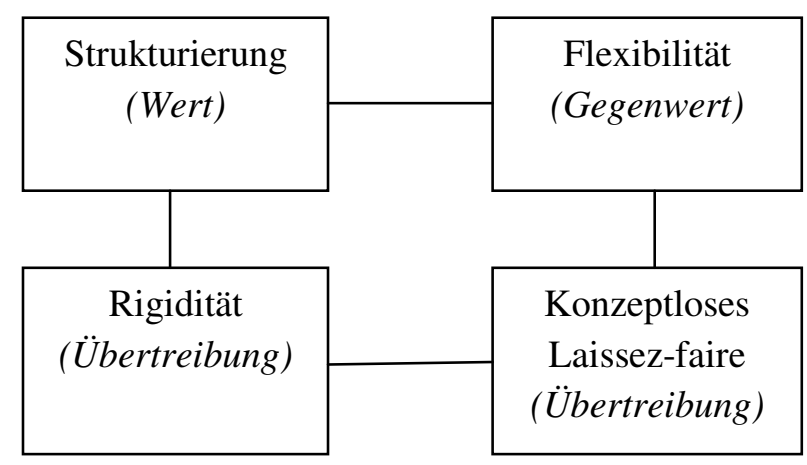

Quelle: eigene Darstellung

Ähnlich ist es mit Dozierenden, die konkrete Vorgaben machen und Regeln setzen. Wird dieses Verhalten nicht gleichzeitig durch die Bereitschaft ergänzt, studentische Autonomie zu fördern und Verantwortung abzugeben, dann entwickelt sich daraus eine negativ zu bewertende Kontrollsucht. Ebenso werden Fürsorge und Verantwortungsübernahme ohne Autonomieförderung zur Über-Behütung. Die Überbetonung von Autonomie und Freiheit hingegen kann zu Leitungs- und Orientierungsverlust und zur Überforderung von Studierenden führen. Beim Umgang mit Konflikten und Regelverstößen gilt es, ein Gleichgewicht zu finden zwischen direkter Konfrontation sowie gegebenenfalls Sanktionierung und einer akzeptierenden, wertschätzenden Haltung gegenüber den Studierenden. Übertrieben konfrontatives Verhalten droht zur Aggression und zur Herabsetzung des Gegenübers zu führen; wird die akzeptierende, wertschätzende Haltung hingegen nicht ergänzt, entwickelt sich daraus Konfliktvermeidung und Nachgeben. Dies sind nur einzelne Beispiele, wie anhand von Wertequadraten die Rolle von Macht in der eigenen Lehre reflektiert werden kann. Das Modell zeigt zudem Entwicklungswege auf, wie eine mögliche Über- oder Unterbetonung von machtorientierten Verhaltensweisen ausgeglichen werden kann (vgl. Sommer 2008). 
Es ist ein dynamischer, nicht ein statischer Balanceakt, der für den angemessenen Umgang mit Macht in der Lehre gefordert ist. Den idealen, fixen Mittelwert, der für alle und in jeder Situation gilt, gibt es nicht. Stattdessen muss die Balance immer wieder gefunden werden. Wo sich das Gleichgewicht einstellt, hängt zum einen von der individuellen Persönlichkeit ab. Von Bedeutung ist ferner die eigene Entwicklung als Lehrender und die der Studierenden. Offenheit, Autonomie und die Übernahme von Eigenverantwortung für Lernprozesse beispielsweise verlangen nicht nur von Studierenden viel ab, sondern erfordern auch auf Seiten der Lehrenden einen souveränen Umgang mit Unsicherheiten und Fehlern (Wörner 2008: 15-22). Zu den Kernkompetenzen machtbewusster Dozierender zählen daher Aufmerksamkeit, Selbstreflexion und Flexibilität. Zielsetzung sollte nicht sein, Macht zu negieren, sondern fair, transparent und wohldosiert mit ihr umzugehen.

\section{Literatur}

Arendt, Hannah 2000: Macht und Gewalt, 14. Auflage, München.

Barnett, Michael/Duvall, Raymond 2005: Power in Global Governance, in: dies.

(Hrsg.): Power in Global Governance, Cambridge, 1-32.

Blom, Herman 2000: Der Dozent als Coach, Neuwied.

Brühl, Tanja/Held, Thomas/Krause, Britta/Lammers, Christiane/Mehl, Regine/ Meyer, Jörg/Nielebock, Thomas/Schmitt, Lars/Schneider, Patricia/Schrader, Lutz 2005: Frieden studieren. Neue Masterstudiengänge für Friedens- und Konfliktforschung an deutschen Hochschulen, Dossier Nr. 48, in: Wissenschaft \& Frieden 1/2005.

Crott, Helmut W. 1997: Macht, in: Frey, Dieter/Greif, Siegfried (Hrsg.): Sozialpsychologie. Ein Handbuch in Schlüsselbegriffen, 4. Auflage, Weinheim, 231-238. French, John P.R./Raven, Bertram 1959: The Bases of Social Power, in: Cartwright, Dorwin (Hrsg.): Studies in Social Power, Institute for Social Research, Ann Arbor, MI, 150-167.

Nielebock, Thomas 2011: Friedens- und Konfliktforschung in der Hochschullehre: Besondere Anforderungen an die Didaktik? Vortrag auf der Jahrestagung des AK Curriculum in Magdeburg, 12.11.2011, unv. Ms.

Schneider, Hans-Dieter (1977): Sozialpsychologie der Machtbeziehungen, Stuttgart.

Schulz von Thun, Friedemann 2008: Miteinander reden, Bd. 2. Stile, Werte und Persönlichkeitsentwicklung, Reinbek bei Hamburg.

Siebert, Horst 2006: Didaktisches Handeln in der Erwachsenenbildung. Didaktik aus konstruktivistischer Sicht, Augsburg. 
Sommer, Angela 2008: Beiträge der Hamburger Kommunikationspsychologie zur Seminargestaltung. Praxisbeispiele und Empfehlungen, in: Berendt, Brigitte/ Voss, Hans-Peter/Wildt, Johannes (Hrsg.): Neues Handbuch Hochschullehre, Griffmarke A 2.3, Berlin.

Spitzer, Manfred 2007: Lernen. Gehirnforschung und die Schule des Lebens, Berlin. Weber, Max 1976: Wirtschaft und Gesellschaft. Grundriss der verstehenden Soziologie, 1. Halbband, 5. revidierte Auflage von Johannes Winckelmann, Tübingen. Wildt, Johannes 2006: Vom Lehren zum Lernen. Zum Wandel der Lernkultur in modularisierten Studienstrukturen, in: Berendt, Brigitte/Voss, Hans-Peter/ Wildt, Johannes (Hrsg.): Neues Handbuch Hochschullehre, Griffmarke A 3.1, Berlin.

Winteler, Adi 2002: Lehrqualität = Lernqualität? Über Konzepte des Lehrens und die Qualität des Lernens (Teil 1), in: Das Hochschulwesen, 50: 2, 42-49.

Winteler, Adi 2005: Professionell lehren und lernen. Ein Praxisbuch, 2. Auflage, Darmstadt.

Witte, Erich H. 2001: Theorien zur sozialen Macht (Hamburger Forschungsberichte aus dem Arbeitsbereich Sozialpsychologie Nr. 30), Hamburg.

Wörner, Alexander 2008: Lehren an der Hochschule: eine praxisbezogene Anleitung, 2. Auflage, Wiesbaden.

\section{Die Autorin}

Dr. Tatjana Reiber, wissenschaftliche Mitarbeiterin, Deutsches Institut für Entwicklungspolitik (DIE)

E-Mail: tatjana.reiber@die-gdi.de 